\title{
O papel do farmacêutico nos serviços de vacinação: revisão integrativa
}

\author{
The pharmacist's role in vaccination services: an integrative review \\ El papel del farmacéutico en los servicios de vacunación: una revisión integradora
}

Recebido: 29/11/2021 | Revisado: 07/12/2021 | Aceito: 10/12/2021 | Publicado: 18/12/2021

\author{
Clarisse Andrade Sales \\ ORCID: https://orcid.org/0000-0002-9451-666X \\ Universidade Federal do Pará, Brasil \\ E-mail: clarisseasales@gmail.com \\ Camila Martins Oliveira \\ ORCID: https://orcid.org/0000-0002-6425-2384 \\ Universidade Federal do Pará, Brasil \\ E-mail: cmofarma@gmail.com \\ Maria Pantoja Moreira de Sena \\ ORCID: https://orcid.org/0000-0001-6641-4618 \\ Universidade Federal do Pará, Brasil \\ E-mail: mariapantojamoreira@ hotmail.com \\ Marcos Felipe Rodrigues de Souza \\ ORCID: https://orcid.org/0000-0002-6246-7525 \\ Universidade Federal do Pará, Brasil \\ E-mail: marcosfrodrigues.br@gmail.com \\ Renato Bruno Cavalcante de Melo \\ ORCID: https://orcid.org/0000-0002-6159-3802 \\ Universidade Federal do Pará, Brasil \\ E-mail: renatobcdemelo@gmail.com \\ Crystyanne de Sousa Freitas \\ ORCID: https://orcid.org/0000-0003-4279-1587 \\ Universidade Federal do Pará, Brasil \\ E-mail: crystyannefreitas@ icloud.com \\ Amanda Gabryelle Nunes Cardoso Mello \\ ORCID: https://orcid.org/0000-0001-7661-1615 \\ Universidade Federal do Pará, Brasil \\ E-mail: agncmello@gmail.com \\ Luann Wendel Pereira de Sena \\ ORCID: https://orcid.org/0000-0001-9363-5766 \\ Universidade Federal do Pará, Brasil \\ E-mail: luannsena@gmail.com
}

\begin{abstract}
Resumo
Objetivo: realizar uma revisão integrativa sobre a atuação do farmacêutico nos serviços de vacinação para que aceitem, reivindiquem e adotem um papel maior na promoção da vacinação no Brasil. Métodos: Trata-se de uma revisão integrativa da literatura sobre a prática dos serviços de vacinação por farmacêuticos. Resultados: Constatou-se que os serviços farmacêuticos estão em crescente evolução, porém, existem poucos estudos que indicam os serviços de vacinação por farmacêuticos no país. Proporcionar altas abrangências vacinais e a inclusão de novas instituições de saúde, como as farmácias e/ou drogarias, vem tornando possível a globalização das vacinações, inclusive a introdução de novas vacinas nos países mais pobres do mundo. O farmacêutico e sua equipe exercem papel fundamental na assistência e/ou cuidado farmacêutico com qualidade. Eles são incumbidos por garantir a execução do acesso a medicamento, como as vacinas, pois tanto o profissional quanto seus auxiliares possibilitam ações que permitem aos usuários compreender melhor sua saúde, seus medicamentos, seu projeto terapêutico e suas necessidades. Conclusão: O professional farmacêutico possui importância significativa, em conjunto com outros profissionais, nos serviços de imunização. No Brasil, este profissional exerce o direito legal e competência técnica para executar esse serviço e a imunização realizada por farmacêuticos nas farmácias e/ou drogarias, além de consolidar a prática clínica, contribuiu para o acesso e aumento das taxas de cobertura vacinais no país.
\end{abstract}

Palavras-chave: Farmacêutico; Serviços clínicos; Vacinação.

\section{Abstract}

Objective: to carry out an integrative review of the role of pharmacists in vaccination services so that they accept, claim, and adopt a greater role in promoting vaccination in Brazil. Methods: This is an integrative literature review on the practice of vaccination services by pharmacists. Results: It was found that pharmaceutical services are in increasing evolution, however, there are few studies that indicate vaccination services by pharmacists in the country. 
Providing high vaccine coverage and the inclusion of new health institutions, such as pharmacies and/or drugstores, has made possible the globalization of vaccinations, including the introduction of new vaccines in the poorest countries in the world. The pharmacist and his team play a fundamental role in providing quality pharmaceutical assistance and/or care. They are responsible for ensuring the implementation of access to medication, such as vaccines, as both the professional and their assistants enable actions that allow users to better understand their health, their medications, their therapeutic project, and their needs. Conclusion: The pharmacist has significant importance, together with other professionals, in immunization services. In Brazil, these professionals exercise the legal right and technical competence to perform this service and the immunization performed by pharmacists in pharmacies and/or drugstores, in addition to consolidating clinical practice, contributed to access and increase vaccination coverage rates in the country.

Keywords: Pharmacist; Clinical services; Vaccination.

\section{Resumen}

Objetivo: realizar una revisión integradora del rol de los farmacéuticos en los servicios de vacunación para que acepten, reivindiquen y adopten un rol mayor en la promoción de la vacunación en Brasil. Métodos: Se trata de una revisión bibliográfica integradora sobre la práctica de los servicios de vacunación por parte de los farmacéuticos. Resultados: Se encontró que los servicios farmacéuticos están en evolución creciente, sin embargo, existen pocos estudios que indiquen servicios de vacunación por parte de farmacéuticos en el país. Brindar una alta cobertura de vacunas y la inclusión de nuevas instituciones de salud, como farmacias y/o droguerías, ha hecho posible la globalización de las vacunas, incluida la introducción de nuevas vacunas en los países más pobres del mundo. El farmacéutico y su equipo juegan un papel fundamental en la prestación de asistencia y/o atención farmacéutica de calidad. Son los encargados de velar por la implementación del acceso a los medicamentos, como las vacunas, ya que tanto el profesional como sus asistentes posibilitan acciones que permitan a los usuarios comprender mejor su salud, sus medicamentos, su proyecto terapéutico y sus necesidades. Conclusión: El farmacéutico tiene una importancia significativa, junto con otros profesionales, en los servicios de inmunización. En Brasil, estos profesionales ejercen el derecho legal y la competencia técnica para realizar este servicio y la inmunización realizada por los farmacéuticos en las farmacias y/o droguerías, además de consolidar la práctica clínica, contribuyó al acceso y aumento de las tasas de cobertura de vacunación en el país.

Palabras clave: Farmacéutico; Servicios clínicos; Vacunación.

\section{Introdução}

As vacinas são agentes imunizadores empregues na prevenção de uma série de enfermidades. São desenvolvidas utilizando-se o próprio organismo causador da doença ou seus derivados, que serão incumbidos por estimular em nosso corpo uma resposta imunológica. Ao receber a vacina, nosso corpo inicia a produção de anticorpos e, graças à nossa memória imunológica, quando tivermos contato novamente com o agente causador da patologia, nossos anticorpos serão processados rapidamente, impedindo, desse modo, que fiquemos doente (Domingues et al., 2019). Por conta disto, vacinar-se é um ato necessário para a proteção individual e coletiva. Por meio dele, algumas doenças já foram erradicadas, como a varíola e a poliomielite. E, apesar de nenhuma vacina ser $100 \%$ eficaz, hoje a imunização é essencial para prevenir óbitos, casos graves da Covid-19 e para conter a pandemia (Castro, 2021).

No Brasil, o símbolo da vacinação se deu no início do século XX, através da política de saneamento e imunização em massa da população, proposta pelo sanitarista Oswaldo Cruz, em 1904, diante do surto de varíola na época. Durante o século $\mathrm{XX}$, ocorreram surtos de outras doenças, como febre amarela, poliomielite e tuberculose, que contribuíram para estabelecer os programas de imunização do governo e a adesão da população às vacinas (Araújo et al., 2019). Em 1973, o governo brasileiro concebeu o Programa Nacional de Imunização (PNI), legitimado pelo Decreto $\mathrm{n}^{\mathrm{o}}$ 78.231/1976, que desde então vem abrangendo diversas vacinas. O PNI tornou-se referência mundial em programas de vacinação, pois conseguiu conter ou até erradicar várias doenças, uma vez que conquistou altas taxas de cobertura vacinal, principalmente nas crianças. O PNI utiliza a maioria dos imunobiológicos produzidos por empresas nacionais, como política de parcerias e incentivo à modernização tecnológica dos produtos brasileiros (Martins et al., 2018).

Há alguns anos os Conselhos Federal e Regionais de Farmácia vem empenhando-se pela regulamentação do serviço de vacinação nas farmácias no Brasil, tanto que, em 2013, o Conselho Federal de Farmácia (CFF) publicou a resolução nº 574, 
que define os direitos e habilidades dos farmacêuticos na dispensação e aplicação de vacinas em farmácias e drogarias (CFF, 2013). Atualmente, essa resolução foi revogada pela Resolução CFF nº 654/2018 (CFF, 2018).

A Lei Federal $n^{\circ} 13.021 / 2014$, que dispõe sobre o exercício e a fiscalização das atividades farmacêuticas, vai ao encontro das ações dos Conselhos de Farmácia, pois indica que as farmácias devem ter assistência farmacêutica (AF) durante todo o período de funcionamento e permite que esses estabelecimentos possuam e apliquem as vacinas (Brasil, 2014). É importante destacar que as farmácias retratam, muitas vezes, o primeiro contato das pessoas ao cuidado em saúde, devido a capilaridade e distribuição geográfica desses estabelecimentos, o que contribui para que o farmacêutico, por sua competência e disponibilidade, possa dispor de serviços que colaborem com a qualidade de vida e promoção da saúde da população (França et al., 2021; Galato et al., 2021).

Em dezembro de 2017, a Agência Nacional de Vigilância Sanitária (ANVISA) publicou a Resolução de Diretoria Colegiada (RDC) $n^{\circ}$ 197, que dispõe sobre os requisitos mínimos para o funcionamento dos serviços de vacinação humana (Anvisa, 2017). Essa norma se aplica a qualquer estabelecimento que faça ou pretenda realizar o serviço de vacinação, sejam eles públicos, privados, filantrópicos, civis ou militares e tem o objetivo de padronizar a prestação do serviço, independentemente da natureza do estabelecimento. Com a finalidade de estabelecer requisitos necessários à prestação do serviço de vacinação pelo farmacêutico, em fevereiro de 2018, o CFF publicou a resolução nº 654 (CFF, 2018; França et al., 2021).

Diante dessa conjuntura, devido a ausência de estudos no Brasil pertinentes ao tema, torna-se fundamental a verificação constante desta temática, no sentido de confirmar a importância do papel do farmacêutico nos serviços de vacinação. Neste sentindo, este estudo teve como objetivo realizar uma revisão integrativa sobre a atuação do farmacêutico nos serviços de vacinação para que aceitem, reivindiquem e adotem um papel maior na promoção da vacinação no país.

\section{Metodologia}

Trata-se de uma revisão integrativa da literatura sobre a prática dos serviços de vacinação por farmacêuticos (Mendes et al., 2019). A triagem dos estudos foi efetuada nas bases de dados Google Scholar, Scientific Electronic, Library Online (SciELO), Medical Literature Analysis and Retrieval System Online (MEDLINE) e Literatura Latino-americana e do Caribe em Ciência da Saúde (LILACS), por meio dos descritores: "vacinação e/ou imunização", "farmacêutico" e "serviços farmacêuticos", nos idiomas português, inglês ou espanhol.

Os critérios de inclusão estipulados para escolha dos artigos foram: artigos que divulgavam texto completo disponível nos idiomas português, inglês ou espanhol, conforme o tema proposto, com livre acesso e publicado nos últimos onze anos. Os artigos de revisão e publicações superiores a onze anos foram excluídos.

Para delineamento da amostra, inicialmente foi processada a identificação dos artigos conforme critérios de inclusão, seguida por escolha baseada na literatura dos títulos e resumos, com posterior eliminação dos estudos que não se enquadrassem na temática da revisão ou que estivessem duplicados nas bases de dados.

Em seguida, foi realizada a análise dos artigos elegíveis com base na leitura integral do seu conteúdo, excluindo-se os artigos não adequados para essa revisão, com posterior seleção final da amostra (Figura 1). 
Figura 1 - Processo de seleção dos estudos.

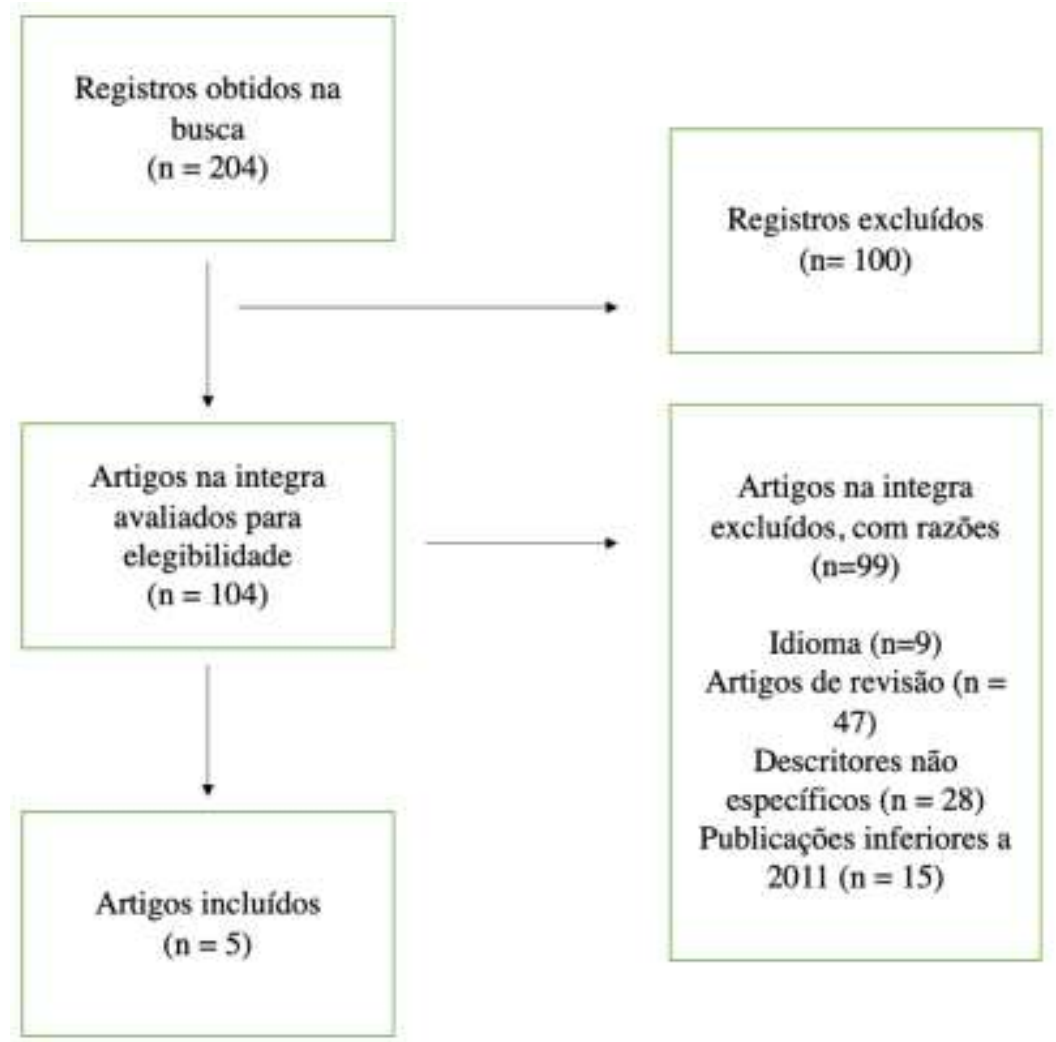

Fonte: Autores.

\section{Resultados}

O processo de seleção dos estudos encontra-se representado na Figura 1. Foram encontrados 204 artigos potencialmente relevantes. Destes, 100 foram excluídos na etapa de triagem. Foram obtidos e lidos na integra 104 artigos, dos quais 9 foram excluídos pelo idioma, 47 porque foi uma revisão sistemática, 28 por não possuírem descritos estabelecidos e 15 devido ao tempo de publicado, superior a onze anos. No final, foram considerados elegíveis 5 artigos científicos (Tabela 1).

Tabela 1 - Descrição dos estudos selecionados.

\begin{tabular}{|c|c|c|c|c|}
\hline $\mathbf{N}^{\mathbf{0}}$ & $\begin{array}{c}\text { Autor (ano)/ } \\
\text { Periódico }\end{array}$ & Título & Tipo de Estudo & Objetivos \\
\hline 1 & $\begin{array}{l}\text { Homma et al. (2011)/ } \\
\text { Ciência \& Saúde } \\
\text { Coletiva }\end{array}$ & $\begin{array}{c}\text { Atualização em vacinas, imunização e } \\
\text { inovação tecnológica }\end{array}$ & $\begin{array}{l}\text { Transversal, } \\
\text { exploratório de } \\
\text { natureza } \\
\text { avaliativa }\end{array}$ & $\begin{array}{c}\text { Demonstrar o fortalecimento, a inovação } \\
\text { tecnológica e modernização da infraestrutura de } \\
\text { produção de vacinas }\end{array}$ \\
\hline 2 & $\begin{array}{l}\text { Pereira et al. (2015) / } \\
\text { Saúde Debate }\end{array}$ & $\begin{array}{c}\text { Serviços farmacêuticos na atenção } \\
\text { primária no município do Rio de Janeiro: } \\
\text { um estudo de avaliabilidade }\end{array}$ & $\begin{array}{l}\text { Transversal, } \\
\text { exploratório de } \\
\text { natureza } \\
\text { avaliativa }\end{array}$ & $\begin{array}{l}\text { Descrever o desenvolvimento de etapas do estudo } \\
\text { de avaliabilidade dos serviços farmacêuticos na } \\
\text { atenção primária à saúde no município do Rio de } \\
\text { Janeiro }\end{array}$ \\
\hline 3 & $\begin{array}{l}\text { Araújo et al. (2017) / } \\
\text { Revista de Saúde } \\
\text { Pública }\end{array}$ & $\begin{array}{l}\text { Atividades farmacêuticas de natureza } \\
\text { clínica na atenção básica no Brasil }\end{array}$ & $\begin{array}{l}\text { Transversal, } \\
\text { exploratório de } \\
\text { natureza } \\
\text { avaliativa }\end{array}$ & $\begin{array}{c}\text { Caracterizar as atividades de natureza clínica } \\
\text { desenvolvida pelos farmacêuticos }\end{array}$ \\
\hline 4 & $\begin{array}{l}\text { Bonadimam et al. } \\
\text { (2018) / Ciência \& } \\
\text { Saúde Coletiva }\end{array}$ & $\begin{array}{c}\text { Nível de satisfação dos usuários e } \\
\text { verificação do conhecimento dos } \\
\text { farmacêuticos em farmácia pública do } \\
\text { Espírito Santo }\end{array}$ & $\begin{array}{l}\text { Descritivo com } \\
\text { abordagem } \\
\text { quantitativa }\end{array}$ & $\begin{array}{c}\text { Determinar o nível de satisfação dos usuários das } \\
\text { farmácias públicas municipais, de dispensação de } \\
\text { medicamentos básicos e verificar o conhecimento } \\
\text { dos farmacêuticos com relação à dispensação } \\
\text { desses medicamentos }\end{array}$ \\
\hline 5 & $\begin{array}{l}\text { Barbosa et al. (2021)/ } \\
\text { Acta Farmacêutica } \\
\text { Portuguesa }\end{array}$ & $\begin{array}{c}\text { Imunização conta a COVID-19: contributo } \\
\text { dos serviços farmacêuticos hospitalares } \\
\text { para o plano de vacinação }\end{array}$ & $\begin{array}{l}\text { Observacional } \\
\text { descritivo }\end{array}$ & $\begin{array}{l}\text { Exemplificar a contribuição do farmacêutico nos } \\
\text { serviços de vacinação }\end{array}$ \\
\hline
\end{tabular}

Fonte: Autores. 
Após escolha e leitura dos artigos indicados, constatou-se que os serviços farmacêuticos (SF) estão em crescente evolução, porém, existem poucos estudos que indicam os serviços de vacinação por farmacêuticos no país. Neste sentido, há necessidade de difundir informações no Brasil, uma vez que, em outros países, esta realidade já está bem estabelecida. Assim, proporcionaremos um maior reconhecimento profissional, auxiliando para o aumento das taxas de cobertura vacinal e consequentemente diminuição do número de mortes por doenças imunopreveníveis.

Um enorme empenho global está sendo realizado para consolidação dos programas de vacinação, especialmente nos países em desenvolvimento. Homma et al. (2011) destaca a necessidade de avanço dos programas de vacinação em todo o mundo. Proporcionar altas abrangências vacinais e a inclusão de novas instituições de saúde, vem tornando possível a globalização das vacinações, inclusive a introdução de novas vacinas nos países mais pobres do mundo.

No propósito de fortalecimento desses programas, grandes esforços têm sido desenvolvidos, em âmbito mundial, para segurar o acesso aos medicamentos. Disparadamente, o consumo e a produção de medicamentos crescem a cada ano, revelando as desigualdades no acesso, o desperdício e o uso inadequado. Essas dificuldades não estão restritas apenas à população geral, mas também envolvem prescritores e outros profissionais de saúde, gerando problemas sanitários, econômicos e sociais para serviços e sistemas de saúde. O farmacêutico e sua equipe exercem papel fundamental na assistência e/ou cuidado farmacêutico com qualidade. Eles são incumbidos por garantir a execução do acesso a medicamento, como as vacinas, pois tanto o profissional quanto seus auxiliares possibilitam ações que permitem aos usuários compreender melhor sua saúde, seus medicamentos, seu projeto terapêutico e suas necessidades (Pereira et al., 2015).

Neste sentido, os SF passaram por uma grande transformação, mudando seu foco do produto para os cuidados com os pacientes, por isso é imprescindível que estes, tenham um entendimento suficiente acerca dos medicamentos, da farmacoterapia, das boas práticas em farmácia, agindo sempre pautado nos princípios éticos. Em seu estudo, Bonadimam et al. (2018) destaca que, a percepção e a satisfação dos pacientes com SF são razões de extrema utilidade para avaliar os serviços oferecidos e, consequentemente, os resultados nos cuidados com a saúde. É importante apontar o conhecimento e a participação dos farmacêuticos na melhoria do sistema de saúde, principalmente na gestão de serviço atingindo na melhoria da qualidade de dispensação de medicamentos.

De acordo com Araújo et al. (2017), as atividades de natureza clínica praticadas por farmacêuticos no Brasil ainda são incipientes. As dificuldades encontradas indicam improvisação e esforço dos profissionais. A reduzida participação em atividades educativas de promoção da saúde aponta pouca integração dos farmacêuticos na equipe de saúde e da assistência farmacêutica (AF) nas demais ações de saúde, inclusive nos serviços de vacinação.

Sabe-se que as vacinas previnem cerca de 2,5 milhões de mortes em todo o mundo a cada ano e estão entre as mais econômicas medidas preventivas contra doenças infecciosas. Apesar da sua eficácia e disponibilidade, as taxas de vacinação e a aceitação deste SF permanecem abaixo do ideal. Barbosa et al. (2021), em seu estudo defende que o processo de vacinação foi contemplado de forma a garantir o acesso igualitário, obedecendo a um planejamento cuidadoso, com priorização de grupos de risco, fases de vacinação e garantia de segurança com minimização de desperdício. O mesmo autor destaca que, desde meados do ano 1800 os farmacêuticos detêm um papel considerável no processo de vacinação, procedendo ao armazenamento de vacinas, à sua distribuição, preparação de antitoxinas e o farmacêutico é o profissional com competência adequadas às exigências deste processo.

Ressalta-se que a realização de métodos que confirmem que as vacinas devem ser armazenadas em local apropriado, dentro do intervalo de temperaturas adequadas, permitindo garantir a capacidade máxima de vida útil da vacina, evitando a sua deterioração e desperdício e a estratégia de vacinação dos profissionais de saúde contou com a farmácia hospitalar na preparação das doses individuais a administrar, obrigando um planejamento adequado às exigências do desafio proposto (Barbosa et al., 2021). 


\section{Discussão}

Os benefícios da vacinação para a saúde pública têm uma grandeza sanitária, social e econômica inquestionável. Conforme a Organização Mundial da Saúde (OMS), ela está antes apenas da água potável. Sendo assim, as vacinas são de relevância para a sustentabilidade e efetividade dos sistemas de saúde, bem-estar dos pacientes, família e comunidades e prosperidade de todos os países (Poudel et al., 2019).

Os serviços de vacinação contra várias doenças mantêm-se aquém daqueles indicados pela OMS e outras agências internacionais. Por mais que, a maioria dos países tenham instituído programas bem-sucedidos e eficazes, ainda há um vasto caminho a percorrer, não apenas para conquistar altas taxas de imunidade do rebanho contra várias doenças, mas também, para obter igualdade no acesso à vacinação ao longo da vida (Vieira et al., 2020).

A OMS apresentou uma campanha para obter $75 \%$ da cobertura vacinal no mundo. A preferência dos usuários pelas farmácias e/ou drogarias para realizarem o procedimento de administração de medicamentos, deveria representar um interesse para considerar a importância de um maior comprometimento desses estabelecimentos na obtenção dessa meta. Dessa forma, o farmacêutico dispõe de uma atribuição essencial na ampliação da cobertura vacinal, possibilitando a diversificação do acesso aos serviços e empregando recursos técnicos-científicos existentes em conjunto com outros profissionais da saúde (Tritany et al., 2020).

A Agência Nacional de Vigilância Sanitária (ANVISA) autorizou a RDC nº 197/2017, que consente a qualquer estabelecimento de saúde, inclusive farmácias e drogarias, comercializar e administrar vacinas, sendo este serviço já previsto pela Lei ${ }^{\circ}$ 13.021/14. O Conselho Federal de Farmácia (CFF), com a finalidade de proporcionar o atendimento com qualidade, de forma acessível aos pacientes, concedeu a RDC nº 654/2018 que, dispões sobre os requisitos necessários à prestação do serviço de vacinação pelo farmacêutico (Anvisa, 2017; Brasil, 2014; CFF, 2018)

A atuação deste profissional na imunização é vinculada à aprovação em curso de formação complementar e à apresentação do documento comprobatório aos Conselhos Regionais de Farmácia (CRF’s) da jurisdição. Os cursos complementares necessitarão, obrigatoriamente, ser credenciado pelo CFF ou oferecido por Instituições de Ensino Superior (IES) reconhecida pelo Ministério da Educação (MEC). Os farmacêuticos que fizerem pós-graduação, obedecendo aos requisitos mínimos previstos na resolução, ou que comprove conhecimento na área de no mínimo um ano, poderão exercer o serviço (CFF, 2018; Galato et al., 2021).

O farmacêutico cumpre um papel fundamental no aumento da conscientização, aceitação e no fornecimento de acesso apropriado aos serviços de vacinação. As farmácias e/ou drogarias, em razão da sua acessibilidade e distribuição geográfica, encontram-se em uma posição ideal para oferecer o serviço em prol do aumento do acesso e/ou cobertura vacinal (Hua et al., 2020).

Em acréscimo ao relato de Pereira et al. (2015), através da vivência, compreensão de doenças e medicamentos (exemplo: vacinas), o farmacêutico também exerce um papel considerável como educadores públicos em saúde, contribuindo assim, para maiores taxas de vacinação (Barbosa et al., 2021). Além disso, colaboram para atenção da resistência microbiana, fortalecimento dos programas de vacinação e maior acesso aos serviços e aos medicamentos. Outrossim, compete também ao farmacêutico, a gestão do uso racional dos medicamentos e outros produtos da saúde, tornando-se necessário na garantia de qualidade e segurança em todas as etapas do processo, desde a resseção, armazenamento, preparação, distribuição, administração e monitorização de efeitos adversos. Assim sendo, este profissional possui habilidades adequadas às exigências deste processo, sempre atendo ao objetivo final: o sucesso do processo de imunização no menor espaço de tempo possível (Poudel et al., 2020; Vieira et al., 2020).

$\mathrm{O}$ atuação do farmacêutico no âmbito da vacinação tem como objetivo possibilitar o serviço, aconselhando a população sobre os seus benefícios, esclarecendo dúvidas e desmitificando concepções erradas, que muitas vezes provocam a 
não adesão à vacinação; reconhecer indivíduos específicos a grupos-alvo e aconselhar a consulta médica, o que é mais facilmente alcançado no decurso do processo de dispensa de medicamentos; dispensar e administrar vacinas a todos os utentes que escolheram a farmácia e/ou drogaria como local para se vacinarem (Patel et al., 2021).

A farmácia e/ou drogaria é tida como a porta de acesso da população em relação ao consumo de medicamentos, devendo ser entendida como um posto avançado de atenção primária a saúde (APS). Segundo dados do Ministério da Fazenda, as farmácias e/ou drogarias seriam responsáveis por 76\% do provimento direto de medicamentos à população (Brasil, 2014). Esses estabelecimentos executam diversos serviços clínicos, ofertado pelo farmacêutico, nos quais empregam seu conhecimento e perícia a fim de melhorar a farmacoterapia e a gestão da patologia, mediante comunicação com o doente ou com outro profissional da saúde, quando necessário (Soares et al., 2020).

Em circunstâncias históricas, a prática farmacêutica vem expandindo sua atividade para além da distribuição dos medicamentos industrializados, há mais de quatro décadas. Em todo mundo, procura-se na atualidade integrar de forma organizada e sistemática esses serviços clínicos aos sistemas de saúde dos países (Soares et al., 2020; Patel et al., 2021).

Ao observar as informações de cada país sobre a atuação do farmacêutico como imunizador, destacam-se o componente legislativo. Com as mudanças nas leis, a ampliação do alcance vacinal foi favorecida, sendo viável ofertar um serviço mais próximo das pessoas, com todos os critérios técnicos-científicos necessários (Gadelha et al., 2020).

$\mathrm{Na}$ Austrália, o provimento de vacinas por farmacêuticos em farmácias comunitárias foi concretizado a partir da publicação sobre a provisão de vacinas pelos farmacêuticos, regulamentada pela Lei de Venenos e Bens Terapêuticos de 1966. Na Nova Zelândia, o governo tem autorizado que os profissionais forneçam vacinas financiadas contra gripe para pacientes acima de 65 anos e mulheres grávidas, além de permitir a vacinação de adultos com menos de 65 anos. Nas Filipinas, a Lei da República, conhecida como Lei da Farmácia de 2016, confirma a vacinação pelos farmacêuticos. Outros países como França, Portugal, Suíça, Irlanda, Canadá, Estados Unidos da América (EUA), realizam os serviços de vacinação por farmacêuticos. Na América do Sul, o processo não é diferente, países como Argentina, Chile, Peru, Venezuela também possuem serviços de vacinação em farmácias e/ou drogarias sendo aplicadas por profissionais farmacêuticos (Homma et al., 2011; Araújo et al., 2019; Barbosa et al., 2021; Castro, 2021).

No Brasil, o serviço de vacinação coordenado por farmacêuticos possui notoriedade, firmando a prática clínica e consolidando as farmácias e/ou drogarias como estabelecimento de saúde. Destaca-se também a importância deste profissional no PNI (Vieira et al., 2021). Esta é uma vitória eminente da profissão farmacêutica no Brasil, pois os farmacêuticos possuem o direito legal e a competência técnica para fornecer e administrar medicamentos com qualidade. Essa experiência foi crucial no processo de desenvolvimento do escopo dos profissionais na prestação de serviços de imunização, seja em farmácias e/ou drogarias, ou, quaisquer outros estabelecimentos de saúde (Freitas et al., 2021).

Entretanto, mesmo com estas grandes conquistas, existem problemas a serem superados, tais como: expandir o número de farmacêuticos credenciados para o fornecimento do serviço de imunização; aumentar o número de cursos que forneçam treinamentos em suporte básico de vida, o que terá um impacto na educação dos farmacêuticos imunizadores; oportunidade para farmacêuticos e farmácias de educarem os pacientes sobre a importância da imunização, com base em dados do Ministério da Saúde Brasileiro (MS), que apontam uma diminuição nas taxas de cobertura de imunização entre adultos e idosos (Noormandi et al., 2021; Varallo et al., 2019).

Contudo, situar o farmacêutico como um imunizador e provedor de serviços na farmácia e/ou drogaria, fortalece as atribuições clínicas do profissional, atribuídas pelo CFF através da Resolução RDC No 585/2013. Proporcionado assim, um maior reconhecimento profissional, adesão aos SF, sustentabilidade e acesso equitativo aos serviços de vacinação (Galato et al., 2021). 


\section{Conclusão}

O farmacêutico possui importância significativa, em conjunto com outros profissionais, nos serviços de imunização. No Brasil, este profissional exerce o direito legal e competência técnica para executar esse serviço. Neste estudo, os resultados demostram que a imunização realizada por farmacêuticos nas farmácias e/ou drogarias, além de consolidar a prática clínica, contribuiu para o acesso e aumento das taxas de cobertura vacinais no país.

Para um domínio maior, recomenda-se que seja pontuado nos próximos estudos, a quantidade de farmácias e/ou drogarias que realizam os serviços de vacinação no Brasil e o perfil de vacinas mais solicitadas pelos usuários.

\section{Referências}

Agência Nacional de Vigilância Sanitária. Resolução RDC no 197, de 26 de dezembro de 2017. Dispõe sobre os requisitos mínimos para o funcionamento dos serviços de vacinação humana. Diário Oficial da União, Brasília, DF, 28 de dezembro, Seção 1, p. 58.

Andreoli G. L. M., \& Dias C. N. (2015). Planejamento e gestão logística de medicamentos em uma central de Abastecimento Farmacêutico hospitalar. Revista de Administração Hospitalar e Inovação em Saúde, 12 (4): 20-28.

Araújo P. S., Costa E. A., Junior A. A. G., Acúrcio F. A., Guibu I. A., Álvares J., Costa K. S., Karnikowski M. G. O., Soeiro O. M., \& Leite S. N. (2017). Atividades farmacêuticas de natureza clínica na atenção básica no Brasil. Revista de Saúde Pública, 51 (1): Supl 2:6s.

Araújo T. M., Souza F. O., \& Pinho P. S. (2019). Vacinação e fatores associados entre trabalhadores da saúde. Cadernos de Saúde Pública, 35 (4): e00169618.

Barbosa R., Fraga S., Sampaio C., Sousa L., Queiroz M., \& Soares P. (2021). Imunização contra a COVID-19: contributo dos serviços farmacêuticos hospitalares para o plano de vacinação. Acta Farmacêutica Portuguesa, 10 (1): 111-115.

Barros, M. E., \& Araújo, I. G. (2021). Avaliação das intervenções farmacêuticas em unidades de terapia intensiva de um hospital de ensino. Revista Brasileira de Farmácia Hospitalar e Serviços de Saúde, 12 (3): 0561.

Bondaiman R. L., Santanna A. F., Brasil G. A., Lima E. M., Lenz D., Endringer D. C., \& Andrade T. U. (2018). Nível de satisfação dos usuários e verificação do conhecimento dos farmacêuticos em farmácias públicas do Espírito Santo, Brasil. Ciência \& Saúde Coletiva, 23 (2): $627-638$.

Brasil. Congresso Nacional Lei $\mathrm{n}^{\circ}$ 13.021, de 8 de agosto de 2014. Dispõe sobre o exercício e a fiscalização das atividades farmacêuticas. Diário Oficial da União, Brasília, DF, 8 de agosto, Seção 1, p.1.

Castro, R. (2021). Vacinas contra a Covid-19: o fim da pandemia? Revista de Saúde Coletiva, 31 (1): e310100.

Conselho Federal de Farmácia (CFF). Farmácia Hospitalar: coletânea de práticas e conceitos. Brasília: CFF, 2017.

Conselho Federal de Farmácia. Resolução n ${ }^{\circ}$ 574, de 22 de maio de 2013. Define, regulamenta e estabelece atribuições e competências do farmacêutico na dispensação e aplicação de vacinas, em farmácia e drogarias. Diário Oficial da União, Brasília, DF, 24 de maio. 2013. Seção 1, p. 181.

Conselho Federal de Farmácia. Resolução no 654, de 22 de fevereiro de 2018. Dispõe sobre os requisitos necessários à prestação do serviço de vacinação pelo farmacêutico e dá outras providências. Diário Oficial da União, Brasília, DF, 27 de fevereiro. 2013. Seção 1, p. 78-79.

Domingues C. M. A. S., Fantinato F. F. S. T., Duarte E., \& Garcia L. P. (2019). Vacina Brasil e estratégias de formação e desenvolvimento em imunizações. Epidemiologia e Serviços de Saúde, 28 (2): e20190223.

Ferranti E. (2017). Gestão de estoque de medicamentos utilizando classificação ABC em um hospital público. Perspectiva Econômica, 13 (3): $215-229$.

França C., \& Andrade L. G. (2021). Atuação do Farmacêutico na Assistência a Saúde em Farmácias Comunitárias. Revista Ibero-Americana de Humanidades, Ciências e Educação, 7 (9): 398-413.

Freitas, G. R., Santos, J. U., França, J. S., Pinto, R. S., \& Seixas, B. V. (2021). Economic impact of pharmacysts' interventions in asthma management: a systemmatic review. Revista Brasileira de Farmácia Hospitalar e Serviços de Saúde, 21 (3):0587.

Gadelha C. A. G., Braga P. S. C., Montenegro K. B. M., \& Cesário B. B. (2020). Acesso a vacinas no Brasil no contexto da dinâmica global do complexo econômico-industrial da saúde. Cadernos de Saúde Pública, 36 (1): e00154519.

Galato D., Alano G. M., Trauthman S. C., \& Vieira A. C. (2021). A dispensação de medicamentos: uma reflexão sobre o processo para prevenção, identificação e resolução de problemas relacionados à farmacoterapia. Revista Brasileira de Ciências Farmacêuticas, 44 (3): 629-640.

Gil R. B., Chaves L. D. P., \& Laus A. M. (2015) Gerenciamento de recursos materiais com enfoque na queixa técnica. Revista Eletrônica de Enfermagem, 17 (1): 100-7.

Gonçalves A. A., Yoshida D., Pitassi C., \& Freitas J. A. S. B. (2016). Fatores críticos de sucesso na gestão da cadeia de suprimentos em saúde pública: uma visão dos gestores dos institutos federais da cidade do Rio de Janeiro, Rj, Brasil. Revista de Administração Hospitalar e Inovação em Saúde, 13 (2): $40-52$.

Hohl., C. M., Partovi N., \& Ghement I (2017). Impact of early in-hospital medication review by clinical pharmacists on health services utilization. PlosOne, 12(2): e0170495. 
Homma A., Martins R. M., Leal M. L. F., Freire M. S., \& Couto A. R. (2011). Atualização em vacinas, imunizações e inovação tecnológica. Ciência \& Saúde Coletiva, 16 (2): 445-458.

Hua X., Gu M. G., Zeng F., Hu H., Zhou T., Zhang Y., \& Shi C. (2020). Pharmacy administration and pharmaceutical care practice in a module hospital during the COVID-19 epidemic. Journal of the American Pharmacists Association, 60 (1): 431-438.

Leite A. L. A. (2019). Gerenciamento da Terapia Medicamentosa: uma análise de indicadores do serviço farmacêutico. Trabalho de Conclusão de Curso, Universidade Federal de Minas Gerais, MG, Brasil.

Maioli N. A., \& Santos H. C. B. (2018). Intervenções Farmacêuticas e sua importância na Segurança do Paciente Hospitalizado. Colloq Vitae, 10 (2): 35-40.

Martins K. M., Santos W. L., \& Álvares A. C. M. (2018). A importância da imunização: revisão interativa. Revista de Iniciação Científica e Extensão, 2 (2): 96-101.

Mendes K. D. S., Silveira R. C. C. P., \& Galvão C. M. (2019). Uso de gerenciador de referências bibliográficas na seleção dos estudos primários em revisão interativa. Revista Texto e Contexto Enfermagem, 28 (1): e20170204.

Menezes K. B., Silva A. S., Cunha-Matos C. E., Nutels T., Oliveira A. D., Neves S. J., \& Leopardi-Gonçalves M. G. (2021). Associação entre discrepâncias medicamentosas e o tempo de internação na clínica cirúrgica em um hospital universitário. Revista Brasileira de Farmácia Hospitalar e Serviços de Saúde, 12 (2): 0547 .

Noormandi A., Karimzadeh I., Mirjalili M., \& Khalili H. (2021). Clinical and economic impacts of clinical pharmacists' interventions in Iran: a systematic review. Daru, 27 (1): 361-378.

Oliveira A. M., Rodrigues V. A. V., \& Passerini J. P. (2018). Queixas técnicas e reações adversas a medicamentos notificadas em um hospital regional no Brasil: um estudo transversal. ABCS Health Sciences, 43 (1): 25-29.

Patel C., Dalton L., Dey A., Macartney K., \& Beard F. (2021). Letter: impact of the COVID-19 pandemic on pharmacist-administered vaccination services. Research in Social and Administrative Pharmacy, 17 (1): 2040-2041.

Pereira N. C., Luiza V. L., \& Cruz M. M. (2015). Serviços farmacêuticos na atenção primária no município do Rio de Janeiro: um estudo de Avaliabilidade. Saúde Debate, 39 (105): 451-468.

Pereira R. M., Felix B. S., Monteiro N. J., \& Fernandes R. M. (2019). Análise da gestão de estoque em uma farmácia hospitalar em Marabá-PA: um estudo de caso. Sistemas \& Gestão, 14 (4): 413-423.

Poudel A., Lau E. T. L., Deldot M., Campbell C., Waite N. M., \& Nissen L. M. (2019). Pharmacist role in vaccination: Evidence and challenges. Vacines, 37 (40): 5939-5945.

Santos N. S., Marengo L. L., Moraes F. S., \& Barberato. (2019). Intervenções para reduzir a prescrição de medicamentos inapropriados para idosos. Revista de Saúde Pública, 53:7.

Silva P. L., Castilho S. R., \& Ferraz C. V. V. G. (2017). Análise dos Resultados da Aplicação de Práticas Gerenciais na Logística de Estoque de uma Farmácia Hospitalar. Revista de Administração Hospitalar e Inovação em Saúde, 14 (2): 14-31.

Silva R. T. D., Santos F. J. S., Bezerril M. S., \& Salvador P. (2020). Estratégias de gestão da qualidade e gestão de material de uma farmácia hospitalar: revisão interativa da literatura. Revista de Administração Hospitalar e Inovação em Saúde, 17 (1): 1983-1991.

Soares L. A., Duarte L. C. S., Morais J. O., Martins J. F., Pereira M. L., Sanches C., \& Baldoni A. O. (2020). Arcabouço legal para implantação e execução dos serviços farmacêuticos relacionados à farmácia clínica. Brazilian Journal of Health and Pharmacy, 2 (4): 26-37.

Tritany R. F., \& Tritany E. F. (2020). Serviços Farmacêuticos no Enfrentamento à COVID-19: Uma revista interativa da literatura. Revista Saúde em Redes, 6 (1): Supl.2.

Valentin A. A. S., Rezende C.P., Nascimento Y. A., Gualberto F. C. M., Mendonça S. A. M., Nascimento M. M. G., \& Oliveira D. R. (2021) Aspectos envolvidos na sustentabilidade do serviço de gerenciamento da terapia medicamentosa. Research Society and Development, 10 (8): e13310817135.

Varallo F. R., Forgerini M., \& Hedeiro M. T. (2019). Harmonization of Pharmacovigilance Regulation in Brazil: Opportunities to improve Risk Communication. Clinical Therapeutics, 41 (3): 598-602.

Varallo F. R., Forgerini M., \& Hedeiro M. T. (2019). Harmonization of Pharmacovigilance Regulation in Brazil: Opportunities to improve Risk Communication. Clinical Therapeutics, 41 (3): 598-602.

Viana S., Arantes T., \& Ribeiro S. (2017). Intervenção do farmacêutico clínico em uma Unidade de Cuidados Intermediários com foco no paciente idoso. Einstein, 15 (3): 283-288.

Vieira E. W., Pimenta A. M., Montenegro L. C., \& Silva T. M. R. (2020). Estrutura e localização dos serviços de vacinação influenciam a disponibilidade do tríplice viral no Brasil. Revista Mineira de Enfermagem, 24 (1): e-1325. 